\title{
Staphylococcus aureus and its IgE-inducing enterotoxins in asthma: current knowledge
}

\author{
Claus Bachert ${ }^{1,2}$, Marc Humbert (10 ${ }^{3}$, Nicola A. Hanania ${ }^{4}$, Nan Zhang ${ }^{1}$, \\ Stephen Holgate ${ }^{5}$, Roland Buhl ${ }^{6}$ and Barbara M. Bröker (10 ${ }^{7}$
}

Affiliations: ${ }^{1}$ Upper Airways Research Laboratory, Ghent University, Ghent, Belgium. ${ }^{2}$ Division of ENT diseases, CLINTEC, Karolinska Institute, University of Stockholm, Stockholm, Sweden. ${ }^{3}$ Service de Pneumologie, Hôpital Bicêtre, Le Kremlin Bicêtre, France. ${ }^{4}$ Section of Pulmonary and Critical Care Medicine, Baylor College of Medicine, Houston, TX, USA. ${ }^{5}$ Clinical and Experimental Sciences, Faculty of Medicine, University of Southampton, The Sir Henry Wellcome Research Laboratories, Southampton General Hospital, Southampton, UK. ${ }^{6}$ Pulmonary Dept, Mainz University Hospital, Mainz, Germany. ${ }^{7}$ Dept of Immunology, University Medicine Greifswald, Greifswald, Germany.

Correspondence: Claus Bachert, Upper Airways Research Laboratory, Ghent University, C Heymanslaan 10, 9000 Gent - Belgium. E-mail: claus.bachertवugent.be

@ERSpublications

Late-onset non-atopic, often severe asthma is not well understood. There is increasing evidence that bacteria and their proteins, specifically $S$. aureus and its superantigens and serine protease-like proteins may represent the triggers we are looking for. http://bit.ly/386oP4G

Cite this article as: Bachert C, Humbert M, Hanania NA, et al. Staphylococcus aureus and its IgE-inducing enterotoxins in asthma: current knowledge. Eur Respir J 2020; 55: 1901592 [https://doi.org/10.1183/ 13993003.01592-2019].

ABSTRACT While immunoglobulin (Ig) E is a prominent biomarker for early-onset, its levels are often elevated in non-allergic late-onset asthma. However, the pattern of IgE expression in the latter is mostly polyclonal, with specific IgEs low or below detection level albeit with an increased total IgE. In late-onset severe asthma patients, specific IgE to Staphylococcal enterotoxins (SE-IgE) can frequently be detected in serum, and has been associated with asthma, with severe asthma defined by hospitalisations, oral steroid use and decrease in lung function. Recently, SE-IgE was demonstrated to even predict the development into severe asthma with exacerbations over the next decade. Staphylococcus aureus manipulates the airway mucosal immunology at various levels via its proteins, including superantigens, serine-protease-like proteins (Spls), or protein A (SpA) and possibly others. Release of IL-33 from respiratory epithelium and activation of innate lymphoid cells (ILCs) via its receptor ST2, type 2 cytokine release from those ILCs and T helper (Th) 2 cells, mast cell degranulation, massive local B-cell activation and IgE formation, and finally eosinophil attraction with consequent release of extracellular traps, adding to the epithelial damage and contributing to disease persistence via formation of Charcot-Leyden crystals are the most prominent hallmarks of the manipulation of the mucosal immunity by $S$. aureus. In summary, $S$. aureus claims a prominent role in the orchestration of severe airway inflammation and in current and future disease severity. In this review, we discuss current knowledge in this field and outline the needs for future research to fully understand the impact of $S$. aureus and its proteins on asthma. 


\section{Introduction}

Asthma is a common airway disease, and mostly develops in allergic individuals before the age of 16 years (early onset), but also may develop later in life in allergic and apparently non-allergic individuals. When compared with early onset allergic asthma, late-onset non-allergic asthma is often more severe. In the early onset group, inhaled allergens usually contribute to the disease development and may contribute to exacerbations, which are also often triggered by viruses [1]. In the late-onset asthma group, the role of allergens is less obvious, even if many patients do have elevated total serum immunoglobulin (Ig)E. In this latter group, chronic rhinosinusitis, especially with nasal polyps (here abbreviated as CRSwNP) is a common comorbidity and considered to aggravate asthma. Typically, specific serum IgE is polyclonal in late-onset asthma, and the single IgE specificities which contribute to the elevated total IgE is often below the detection level. Identifying possible stimuli of this IgE response is demanding, but likely important for the management of asthma.

Asthma phenotypes are generally categorised as allergic (atopic) or non-allergic (non-atopic or intrinsic) [2]. The defining feature of allergic asthma is the patient's immune reaction to common inhalant allergens that can be diagnosed by in vitro tests, skin prick tests or airway exposure (provocation tests). Allergic asthma is typically driven by $\mathrm{T}$ helper (Th) 2 cell-mediated inflammation, resulting in the production of cytokines, such as interleukin (IL)-4, IL-5, IL-9 and IL-13. B-cell activation triggers the release of allergen-specific IgE antibodies. The resulting type 2 inflammation is associated with a number of consequences, including airway eosinophilia, mast cell and basophil degranulation, airway smooth muscle contraction, epithelial cell dysfunction and mucus hypersecretion.

In contrast, patients with non-allergic eosinophilic asthma lack allergic reactions to common aeroallergens, reflected by a negative skin prick tests to aeroallergens and the absence of circulating specific IgE [3]. However, similar to atopic asthma, the majority of patients show exaggerated type 2 inflammation in their airways and demonstrate elevated total serum IgE levels [4-6]. A negative skin prick test in non-atopic asthma may occur because the patient has been sensitised to untested or unknown allergens $[3,7,8]$. Alternatively, it has been hypothesised that non-allergic eosinophilic asthma can be predominantly mediated by type 2 innate lymphoid cells (ILC2s), directly driving B-cell activation and class switch to IgE in an antigen-independent manner [2,9]. In any case, patients with non-allergic asthma share many features of the inflammatory response with their allergic counterparts [4].

Chronic rhinosinusitis, long-term inflammation of the nasal and paranasal sinuses, also needs to be discussed in this context because of its role as a frequent comorbidity. The disease is categorised based on the presence or absence of nasal polyps: CRSwNP and chronic rhinosinusitis without nasal polyps (CRSsNP) [10]. Nasal polyps are predominantly characterised by type 2 (eosinophilic) inflammation, whereas CRSsNP is predominantly a non-type 2 (neutrophilic) disease [10, 11]. Type 2 nasal polyps are rarely seen in children or adolescents and mostly develop between 35 and 55 years of age. Nasal polyps are associated with late-onset asthma, which may precede nasal polyps or may be diagnosed later. Type 2 inflammation predicts asthma comorbidity in nasal polyp patients, with the asthma also demonstrating type 2 features [11]. About $20 \%$ of subjects with nasal polyps and asthma additionally report exacerbations with aspirin and other non-steroidal anti-inflammatory drugs (NSAIDs), which are also characterised by a type 2 immune reaction, and are phenotyped as aspirin- or NSAID-exacerbated respiratory disease (AERD or N-ERD) [12]. Aspirin induces specific changes in the arachidonic acid metabolism of those sensitive patients. A common feature of allergic, non-allergic and N-ERD patients with nasal polyps are increased numbers of eosinophils and total serum IgE levels and polyclonal IgE formation within the polyp tissue. Clinical studies have suggested a functional role of IgE in severe asthma and nasal polyps because it was observed that the anti-IgE monoclonal antibody, omalizumab, was effective in airway diseases in allergic and non-allergic subjects $[13,14]$.

Here, we propose that allergic and non-allergic asthma may be more similar than assumed, not only in terms of the eosinophils involved, but also in terms of functional IgE antibodies directed to inhalant and other allergens. A subpopulation of patients classified as non-allergic may in fact be allergic to as yet undiagnosed or unknown allergens, while allergic asthma may also be facilitated or exacerbated by those non-specific stimuli assumed to cause non-allergic asthma.

In a recent study in patients with severe asthma previously diagnosed as non-allergic using a limited number of allergens, at least 50\% showed reactions to other allergens when a specific IgE antibody binding to a broad panel of substances was measured [8]. Proteins of Staphylococcus aureus were among the most frequent so far undiagnosed allergens revealed in this study. They are the focus of our review.

S. aureus, a Gram-positive bacterium, is a common human coloniser, which, given the opportunity, can turn into a dangerous pathogen $[15,16]$. In humans, the upper airways, especially the anterior nares, and the skin are the main sites of $S$. aureus colonisation $[17,18]$. The complex and versatile micro-organism is 
capable of releasing a broad spectrum of virulence factors, including enzymes and toxins. In humans, most extracellular $S$. aureus proteins elicit predominantly type 1 and type 3 immune responses characterised by the release of interferon (IFN) $-\gamma$ and IL-17 as well as the production of specific IgG [19-22]. A small subgroup of $S$. aureus extracellular components, however, are IgE-reactive: the staphylococcal enterotoxins $(\mathrm{SE})$, the serine protease-like proteins (Spls), fibronectin-binding protein (FnBP) as well as extracellular vesicles (EVs) [23-25]. Being a common coloniser of the upper airways, S. aureus has been implicated in aggravating allergy and inducing IgE formation. An increased prevalence of $S$. aureus colonisation as well as sensitisation against its proteins is found in atopic dermatitis, nasal polyps and asthma [26, 27].

The aim of our review is to summarise current evidence for a role of $S$. aureus as tissue-localised modifier of airway disease and discuss the mechanisms by which $S$. aureus can promote airway disease. We also describe the possible role of specific IgE to SEs as a biomarker in nasal polyp disease and asthma, and discuss other sources of $S$. aureus proteins in the environment outside the airways. Finally, we highlight open questions and unmet research needs to fully understand the possible impact of this germ and its products on asthma.

\section{S. aureus and its products in the environment}

$S$. aureus is robust and can survive hostile environmental conditions. Of all the microbial components present in indoor dust, including Gram-positive and Gram-negative bacteria, Pseudomonas spp., Acinetobacter spp., Enterobacter spp. and Staphylococcus spp. are the dominant genera [28]. The presence of $S$. aureus and its allergenic components in house dust has been associated with allergic diseases such as asthma. In a recent study evaluating the prevalence of environmental sEs in households of adolescents with asthma, exposure to detectable levels of $\mathrm{SE}$ proteins $(\mathrm{SEA}-\mathrm{SEF})$ in house dust was frequent, ranging from $36 \%$ ( $\mathrm{SEB}$ and $\mathrm{SEC}$ ) to more than 60\% (SEA) [29]. The presence of $S$. aureus and $S$. aureus-derived extracellular vesicles (EVs) in house dust was also reported; these EVs in indoor dust can evoke inflammatory lung disease, including asthma [28]. The house dust also typically contains house dust mites (HDMs) as a potent source of allergens, and their microbiomes contain a wide variety of Gram-negative and Gram-positive bacteria, including S. aureus and Escherichia coli [28]. The mites thus act as potential carriers for IgE-reactive bacterial antigens, and sensitisation to HDM is common in patients suffering from respiratory problems [30]. Dzono et al. [30] demonstrated the presence of bacterial antigens in purified HDM bodies using specific antibody probes and verified that HDM contain IgE-reactive bacterial antigens by qualitative IgE immunoblot and quantitiative ImmunoCAP inhibition experiments. Additionally, in a small study in 24 homes, $50 \%$ of bedrooms were found to be contaminated with $S$. aureus based on culture for detection, but when real-time PCR methods for detection and quantification of S. aureus (femB) were used, the micro-organism was discovered in $96 \%$ of the bedrooms. Prevalence and SE gene detection in bedroom dust were 21 to $63 \%$ for $\mathrm{SEA}, \mathrm{sEB}, \mathrm{SEC}$ and $\mathrm{sED}$, respectively [31].

\section{S. aureus and its products in the airways}

In humans, the anterior nares are the main site of colonisation for S. aureus; however, the bacterium may also colonise other parts of the body, such as the skin of the axilla, groin, chest/abdomen, as well as the pharynx, intestine and perineum $[17,18]$. The prevalence of $S$. aureus nasal carriage varies considerably with geographical location. In Europe and the USA, approximately $20 \%$ of the healthy population are permanent carriers of $S$. aureus (carry a single strain most of the time), 30\% are intermittent carriers (strains change with varying frequency) and $50 \%$ are non-carriers (no evidence of $S$. aureus despite repeated sampling) [18, 32]. A meta-analysis of five general population-based studies from different geographical locations, including America, Europe, and the Middle East, reported an overall mean prevalence of nasal S. aureus colonisation of around 30\% [33]. Patients with airway diseases, such as asthma and nasal polyps, show significantly higher rates of $S$. aureus colonisation than healthy adults [25]. The bacterium can be found colonising the airways in up to $90 \%$ of patients with nasal polyps, with the highest prevalence in patients with comorbid asthma and aspirin sensitivity. In CRwNP patients, S. aureus grows intramucosally and even intracellularly $[34,35]$. More than 600 proteins released by $S$. aureus were identified in the upper airway mucosa of patients with CSRwNP by high resolution mass spectrometry; among these were SEs and Spls [36].

\section{S. aureus-specific IgE in severe asthma}

In accordance with the increased colonisation rates, the formation of serum IgE against staphylococcal enterotoxins ( $\mathrm{SE}-\mathrm{IgE}$ ) is also increased in patients with chronic airway diseases compared with the general population [27, 34]. In nasal polyps, type 2 inflammation characterised by IL-5 and SE-IgE expression is related to the severity of the local mucosal inflammation and also significantly associated with asthma comorbidity [37,38]. Numerous studies show that SE-IgE-positive individuals have an increased risk of asthma. Moreover, in asthmatics, $\mathrm{SE}-\mathrm{IgE}$ is associated with more severe disease [39]. In the general adult 
European population (>2900 subjects in 19 centres), SE-IgE was significantly associated with asthma (OR 2.10, 95\% CI 1.60-2.76; $\mathrm{p}=0.001$ ) in a concentration-dependent manner [26]. Logistic regression analyses in a British asthma cohort demonstrated significantly increased risks for SE-IgE positive subjects to have any asthma (OR 7.25, 95\% CI 2.7-19.1) or severe asthma (OR 11.09 95\% CI 4.1-29.6) versus negative subjects [23]. In contrast, the presence of grass pollen or house dust mite specific IgE antibodies was not associated with either increased risk for asthma or asthma severity. SE-IgE in asthmatics was indicative for a significantly increased oral steroid use, more frequent hospitalisations within the last year, and a lower lung function (forced expiratory volume in $1 \mathrm{~s}$ (FEV1) \% predicted value). SE-IgE positivity in serum was significantly more frequent in patients with severe asthma than in healthy control subjects (60 versus 13\%; $\mathrm{p}<.001)$. Furthermore, $21 \%$ of the patients with severe asthma and SE-IgE were otherwise non-atopic, i.e. had only SE-specific IgE but no IgE response to common inhalant allergens. Another study confirmed that SE-IgE could be found in atopic and non-atopic patients; in non-atopic asthma, total serum IgE levels were significantly increased in patients sensitised to SES compared with patients non-sensitised to SES. Out of 224 patients with non-atopic and/or late-onset asthma, 105 (47\%) patients were sensitised to SES [40]. The study concluded that SE sensitisation may contribute to Th2-mediated inflammation in non-atopic patients.

In adolescents, the presence of SE-IgE in serum has been also been associated with polyclonal $\operatorname{IgE}$ and multimorbidity in terms of airway disease [41]. Corroborating the European studies, Song et al. [42] reported similar observations from Korea. Serum SE-IgE concentrations were significantly higher in Korean patients with asthma than in controls [42]. Elderly asthma patients with high SE-IgE levels had more severe asthma, sputum eosinophilia and chronic rhinosinusitis compared with those with lower SE-IgE levels. In multivariate logistic regression analyses, the association between serum SE-IgE concentrations and severe asthma was significant, independently of co-variables (OR 7.47, 95\% CI 1.86-30.03; p=0.005). Multiple correspondence analyses also showed that high serum SE-IgE level had close relationships with severe asthma, sinusitis and sputum eosinophilia all together [42]. This was the first report on significant associations of SE-IgE sensitisation with late-onset asthma in the elderly, particularly severe eosinophilic asthma with CRS comorbidity.

The IDENTIFY study gathered data on sensitisation towards aeroallergens in patients with severe asthma, in whom no allergen could be detected in previous routine tests and who are therefore considered to have non-atopic asthma [7]. In the non-atopic asthma group $(n=188)$, allergic sensitisation to at least one allergen was additionally detected in $52 \%$ of patients using an extended panel of aeroallergens. The most frequent sensitizations found were to $\mathrm{SEB}(\sim 25 \%)$ and $\mathrm{seA}(\sim 15 \%)$, and $8 \%$ of the patients were mono-sensitised to SEA and/or SEB. This still may be an underestimation of sensitisations to $S$. aureus SES, as the species harbours 23 superantigen genes, of which only two have been tested. This suggests that in addition to standard perennial allergens, other allergens including SEs should be tested, specifically in those patients who have "non-atopic" asthma. The exact consequences of these sensitizations require additional studies.

Similarly, in a small study comparing COPD patients with controls, SE-IgE positivity was four times higher in both stable and exacerbated COPD subjects compared to healthy controls, either non-smokers or smokers, and SE-IgE serum concentrations were significantly higher in both COPD groups versus controls. SE-IgE decreased significantly during hospitalisation in the exacerbated patients going along with a significant increase in $\mathrm{FEV}_{1}$. Thus, similar to severe asthma, SE-IgE may be relevant in a subgroup of COPD patients [43].

\section{Serum SE-IgE predicts severe asthma}

In a nested case-control study from the 20-year Epidemiological study on the Genetics and Environment of Asthma (EGEA) cohort, including 225 adults (75 without asthma, 76 with mild and 75 with severe asthma) in EGEA2 (2003-2007), SE-sensitisation was measured on samples collected 11 years earlier (EGEA1) [44]. Cross-sectional associations were conducted for both, EGEA1 and EGEA2, and longitudinal analyses estimated the association between SE-sensitisation in EGEA1 and the risk of severe asthma and asthma exacerbations assessed in the follow-up. Models were adjusted on sex, age, smoking, parental asthma/allergy and skin prick test to house dust mite. SE-sensitisation varied between $39 \%$ in controls to $58 \%$ and $76 \%$ in mild and severe asthma in EGEA1, respectively. SE-sensitisation was associated with an increased risk for severe, but not for mild asthma, clearly supporting former findings of an independent cohort [23]. Furthermore, sE-sensitisation in EGEA1 was associated with severe asthma (adjusted-OR 2.69, 95\% CI 1.18-6.15) and asthma exacerbations (adjusted-OR 4.59, 95\% CI 1.40-15.07) assessed 10 to 20 years later. Thus, for the first time, this study showed that being sensitised to SES is associated with an increased subsequent risk of severe asthma and asthma exacerbations. Inhalant allergens 
did not show such association, making SE-IgE a possibly unique predictor of natural course of disease development.

\section{How could S. aureus trigger or exacerbate type 2 airway inflammation?}

S. aureus has numerous means of promoting type 2 airway inflammation. These are assumed to represent immune escape mechanisms whose effectiveness is underlined by the increased prevalence of $S$. aureus colonisation in allergic and type 2 inflammatory patients [25]. The bacteria release $\alpha$-toxin, highly damaging to epithelial layers and presumably facilitating allergen entry [45]. NAKAMURA et al. [46] reported that $\delta$-toxin can trigger mast cell degranulation independent of IgE. SES and other superantigens (see below) can induce an excessive cytokine release from tissue T cells, including Th2 cytokines such as IL-5, IL-4 and IL-13, but they can also induce the rapid release of typical mast cell mediators when specific $\mathrm{SE}-\mathrm{IgE}$ is present [47, 48]. In cultures of fresh nasal polyp tissue with intramucosal $S$. aureus, staphylococcal proteins can be measured in increasing concentrations over $24-72 \mathrm{~h}$ paralleled by the spontaneous release of IL-5 [49]. Killing the intramucosal micro-organisms in the tissue by antibiotics or specific bacteriophages significantly reduces the release of its proteins and suppresses IL-5 production. Recent findings on the migration of eosinophils through the human airway epithelium in response to $S$. aureus exposure, releasing eosinophil extracellular traps (EETs) to immobilise the bacteria, add another aspect to the multifaceted role of $S$. aureus in type 2 inflammation [50]. The IL-5-activated eosinophils may be instrumentalised by $S$. aureus to further break down the epithelium by inducing the release of major basic protein, an epithelium-toxic constituent of eosinophil extracellular traps. With the release of EETs, galectin 10 is released into the epithelial layer, forming Charcot-Leyden crystals (CLCs), which further contribute to maintain the persistent inflammatory response [51].

\section{Promoting functional IgE formation}

A prominent group of $S$. aureus exotoxins, including the SES, act as superantigens (see below). In Th2 cells these will induce the release of IL-4 and IL-13, which are known to promote class switching to IgE and IgE formation. Both cytokines can be found increased in nasal polyps together with increased levels of $\mathrm{B}$ cell activating factor (BAFF) supporting B cell activation [52], and in the inflamed airway tissues IgE-positive B cells, soluble IgE as well as follicle-like structures have been demonstrated [14]. SEB facilitates sensitisation to ovalbumin and consecutive bronchial inflammation with features of allergic asthma in mice, likely due to augmentation of dendritic cell migration and maturation as well as allergen-specific $\mathrm{T}$ cell proliferation [53]. Besides the numerous $\mathrm{T}$ cell superantigens, S. aureus also produces a B cell superantigen, the staphylococcal protein A ( $\mathrm{ppA})$. SpA can activate all B cells that use a $\mathrm{V}_{\mathrm{H}} 3$ element in the $\mathrm{B}$ cell receptor $[54,55]$. It is plausible that $\mathrm{T}$ cell superantigens can also act as "super-allergens" providing help to pre-existing B cells in an antigen non-specific manner, thereby stimulating IgE responses directed against unrelated allergens [53]. The pleiotropic effects of T cell- and B cell superantigens is discussed below.

It needs to be clarified whether $S$. aureus, besides boosting type 2 inflammation via non-antigen-specific mechanisms, can also produce allergens, i.e., antigens which typically induce specific allergic responses in susceptible individuals [56]. Many humans have S. aureus-reactive type $2 \mathrm{~T}$ cells as well as IgE that binds to $S$. aureus factors, indicating that the micro-organism is capable of producing allergens $[25,57]$.

\section{Role of Spls}

The majority of clinical S. aureus isolates can release Spls, a group of up to six proteins, Spls A-F, which are encoded in one operon $[58,59]$. Remarkably, the human $\mathrm{T}$ cell response to Spls is characterised by IL-4, IL-5 and IL-10, while IFN- $\gamma$ - and IL-17 production is very low. This is in strong contrast to the IFN- $\gamma$ - and IL-17-dominated immune reactions typically elicited by other $S$. aureus antigens [20, 21, 26]. Moreover, the majority of asthma patients and a minority of healthy adults have Spl-specific serum IgE. In a mouse model, repeated intra-tracheal administration of SplD triggered allergic lung inflammation characterised by bronchial hyper-responsiveness, strong eosinophilic infiltration in the lung tissues and bronchoalveolar lavage fluid (BALF), and $T$ cell accumulation in the BALF [26]. This allergic inflammation is dependent on IL-33 as well as on T cells. It is accompanied by the generation of SplD-specific IgE and IgE antibodies to other antigens simultaneously administered, and the development of Th2 cells, which are found in the lung draining lymph nodes. Interfering with IL-33 receptor binding with soluble ST2 will decrease most of the type 2 immune reactions, such as the numbers of eosinophils and IL-5/IL-13 production by lymph nodes [24].

S. aureus may also directly contribute to the type 2 immune reaction via the release of IL-33, triggering ST2 positive ILC2s and Th2-cells $[60,61]$. Interestingly, significantly increased numbers of ILC2s can be found in nasal polyps in patients with comorbid asthma, supporting the pathogenic role of IL-33 [62]. Human ILC2s, driven by GATA3 [63], can produce at least small amounts of IL-4 [64-67], and the 
stimulation of adult human ILC2s with IL-33 in combination with IL-2 or IL-7 induces release of IL-4 [68]. This may result in increased IgE production without antigen specificity, comprising hundreds of functional IgE specificities and contributing to often very high total tissue IgE levels [48], along with pronounced eosinophilic inflammation (type 2 inflammation) [50, 69] The functionality of SE-IgE antibodies from nasal polyp tissue has been repeatedly demonstrated, using mast cell and basophil activation or the promotion of IgE-facilitated allergen binding to B cells (IgE-FAB) [48, 70]. Interestingly, in nasal polyps, high concentrations of both IgE and IgG antibodies can be found. IgG antibodies are able to limit IgE-mediated inflammation by competition, because IgG repertoires widely share the antigen targets with the IgE repertoires in both allergic and non-allergic subjects. This resembles the working principle of allergen-specific immunotherapy in allergic subjects, which elicits blocking IgG antibodies that bind the allergens before cell-bound $\operatorname{IgE}$ can do so [71].

\section{SES and other T cell superantigens}

$\mathrm{T}$ cell superantigens (SAgs) are well studied virulence factors of $S$. aureus, which have been implicated in allergy. The species harbours $23 \mathrm{SAg}$ genes encoding the staphylococcal enterotoxins or enterotoxin-like proteins A to $U$ and toxic shock syndrome toxin 1 (TSST-1) [72-74]. The molecules are structurally related but of limited sequence similarity. The equipment with SAg genes as well as their regulation is highly variable as many of them are encoded on mobile genetic elements. Around $80 \%$ of clinical $S$. aureus isolates harbour SAg genes, on average 5-6 [75]. SAgs are exceptionally stable and have remarkable effects on the immune system. They can circumvent the $\mathrm{T}$ cells' requirement for antigen recognition by directly crosslinking $\mathrm{T}$ cell receptors using specific V $\beta$ elements with major histocompatibility complex (MHC) class II molecules, e.g. on dendritic cells or B cells, thereby activating large proportions of T cells irrespective of their antigen specificity (figure 1a) $[72,73]$. Some SAgs also are known to cause food poisoning by inducing vomiting and diarrhoea. These are called SES, the others SE-like [76]. Apart from their function as SAgs, the molecules are also conventional antigen targets for the immune system. In fact, they are strongly immunogenic, and adults have a SAg-neutralising IgG response, which in S. aureus carriers is specific for the colonising strain (figure 1b) [77]. As described above, patients with allergic diseases often develop SAg-specific IgE antibodies (SE-IgE; figure 1b), suggesting that SES may also function as allergens [78]. SE-IgG can be measured in serum using commercially available tests for IgE binding to SEs A-E and TSST-1 (SE-IgE). Whether IgE responses are also directed against the other 17 SAgs is not

Staphylococcal enterotoxins (SEs) acting as:

a) T-cell superantigen

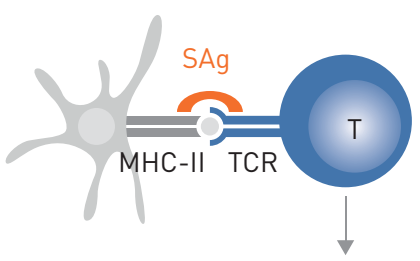

Polyclonal

T-cell activation b) Conventional antigen

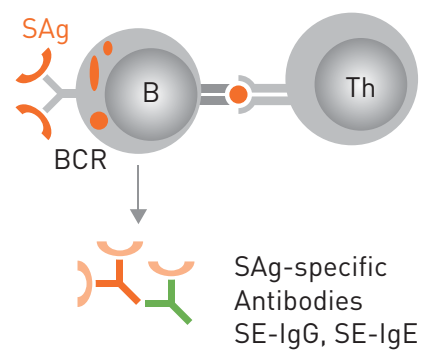

Staphylococcal protein A (SpA) acting as:

c) B-cell superantigen

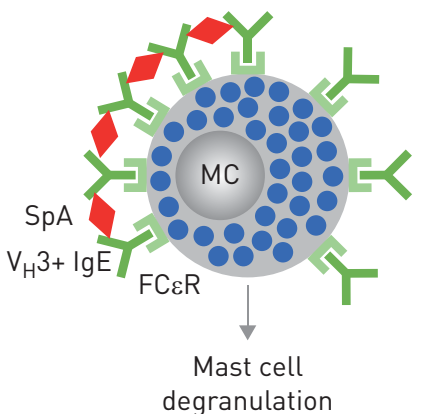

FIGURE 1 Possible functions of staphylococcal T cell and B cell superantigens in allergy. Staphylococcal enterotoxins (SES) and other T cell SAgs can act as a) T cell superantigen: SAgs have superantigen activity and can circumvent conventional antigen processing and peptide presentation by directly cross-linking conserved areas of the T cell receptor (TCR) with areas on major histocompatibility complex (MHC) class II molecules outside the peptide binding cleft. This leads to the polyclonal activation of T cells, including T helper (Th)2 cells, independent of their antigen specificity. b) Conventional antigen: antigen-presenting cells (in this case a SAg-specific B cell) can handle SAgs as conventional antigens: They internalise and process them into peptides, which are then presented to specific T cells on MHC class II molecules. Th cells binding these peptide-MHC complexes with their specific TCRs will provide help for the B cells. The B cells differentiate and produce SAg-specific antibodies. Staphlococcal protein A (SpA) can act as c) B cell superantigen: SpA can bind to $\mathrm{V}_{\mathrm{H}} 3$-positive immunoglobulins. If $\mathrm{V}_{\mathrm{H}} 3$-positive immunoglobulin (lg)E is bound to a mast cell via its $F c \varepsilon$ receptor, SpA will mimic allergen binding and induce degranulation. APC: antigen presenting cell; B: B cell; BCR: B cell receptor; FceR: Fce receptor; MC: mast cell; MHC: major histocompatibility complex; SAg: T cell superantigen; SpA: staphylococcal protein A; T: T cell; Th: T helper cell; TCR: T cell receptor, $V_{H}$ : variable element of the immunoglobulin heavy chain. 
known. As indicated, SE-IgEs are functional, degranulate mast cells and basophils, and are, like inhalant allergens, partially inhibited by IgG antibodies due to overlapping repertoires [70]. This recent publication based on findings in nasal polyps demonstrated: the local specific IgE antibodies in nasal polyp homogenates from patients with CRSwNP are functional and promote FceRI- and FceRII-mediated allergic responses; IgG-associated inhibitory responses are based on the fact that antibody repertoires are widely shared by IgG- and IgE-producing B cells; and local SE-specific IgG antibodies are also induced and can suppress SE-IgE and FceRII-mediated facilitated allergen presentation by B cells to T cells.

Findings from this study suggest that IgE antibodies including SE-IgEs are functional in patients with CRSwNP and comorbid asthma, and that the IgG repertoire is regulating IgE-mediated inflammatory responses, representing a natural blocking mechanism as observed in allergen specific immunotherapy. Besides total IgG, specifically IgG4 is known to block IgE function and cannot bind to FcRs that are present on mast cells [79]; especially S. aureus derived Spls are strong IgG4-inducers [80] and thus could exert a strong IgE blocking activity.

\section{SpA: a B cell superantigen}

Almost all S. aureus strains express $\mathrm{SpA}$, a $45 \mathrm{kDa}$ multi-domain protein with pleiotropic functions in pathogen-host interaction [81]. Protein A is produced by clinical $S$. aureus isolates in varying amounts and anchored in the bacterial cell wall, from where it can be released by proteolytic cleavage. The spa gene contains repetitive sequence motifs that are highly variable and can be used for $S$. aureus molecular typing [82]. The protein SpA is best known for its ability to bind to the Fc-domain of human IgG, interfering with IgG Fcy receptor binding and downstream functions, importantly opsonophagocytosis [81]. Furthermore, SpA can function as a $\mathrm{B}$ cell superantigen by crosslinking B cell receptors that use $\mathrm{V}_{\mathrm{H}} 3$ elements. $83 \mathrm{SpA}$ can bind to many different $\mathrm{V}_{\mathrm{H}} 3$-positive immunoglobulins but not to immunoglobulins of the other six $\mathrm{V}_{\mathrm{H}}$ families [83]. In humans, S. aureus infection SpA skews the antibody response: $\mathrm{V}_{\mathrm{H}} 3+$ plasmablasts are over-represented in the peripheral blood, and these predominantly bind to SpA but not to other S. aureus antigens [84]. In mice, SpA-neutralising antibodies, induced by active vaccination or passively transferred, increase the antibody response to a broad spectrum of $S$. aureus virulence factors. This improves protection from $S$. aureus infection as well as clearance of colonising $S$. aureus [85, 86]. Both $S$. aureus cells and soluble $\mathrm{SpA}$ can also bind to $\mathrm{V}_{\mathrm{H}} 3$-positive IgE antibodies. Mast cells which are decorated with such antibodies via their Fce receptors are then triggered to degranulate (figure 1c) [83, 87]. To explain the high local IgE concentrations in CRSwNP, it has been proposed that SpA can induce IgE production in $\mathrm{V}_{\mathrm{H}}$ 3-positive $\mathrm{B}$ cells [88-91]. Experiments aiming to induce IgE secretion in human $\mathrm{B}$ cells with soluble SpA or S. aureus cells in cell culture were partially successful so far [92-94].

\section{Clinical implications in asthma and it comorbidities}

S. aureus is a frequent opportunistic pathogen, playing a prominent role as infectious agent in human disease. In this situation, the pathogen elicits a neutrophilic response driven by type 1 and 3 immune reactions, interferons and IL-17. In addition, evidence is accumulating that $S$. aureus may have another so far underestimated role in type 2 immune reactions, specifically in atopic dermatitis, nasal polyps (CRSwNP) and asthma [95]. There, the germ may aggravate the inflammation contributing to IgE formation and eosinophil activation (figure 2). In those conditions, S. aureus is found accumulating at sites of disease, as the type 2 immune response module counteracts the protective type 1 and type 3 response modules, giving the microorganism a survival advantage $[25,96]$. In CRSwNP, staphylococcal proteins such as SEs, SE-like SAgs and Spls have been identified within the inflamed tissue, which, when added to the human mucosal tissues ex vivo, evoke strong pro-inflammatory and type 2 deviating immune reactions [36, 97]. Furthermore, the bacteria themselves colonising the airway mucosa, as well as Spls released from $S$. aureus, induce epithelial alarmins, specifically IL-33, which consecutively activates ILC2s and Th 2 cells $[24,60]$. S. aureus also produces a B-cell superantigen, $\mathrm{SpA}$, which can activate all $\mathrm{B}$ cells that use a $\mathrm{V}_{\mathrm{H}} 3$ element in their $\mathrm{B}$ cell receptor [81]. We propose that in an IL-4 and IL-13 rich mucosal environment, also expressing BAFF and follicle-like structures, the B-cell superantigen may induce polyclonal IgE and high total IgE production, as it is typically seen in severe CRSwNP, asthma and atopic dermatitis. Innovative ways of interference with these triggers, such as immunisation against $S$. aureus proteins or IL-33 receptor inhibition, may be offering relief in severe disease atopic dermatitis, asthma and CRSwNP in the future, besides conferring protection from $S$. aureus colonisation by restoration of the epithelial barrier.

Furthermore, IgE with specificity for staphylococcal SAgs (SES) is formed within the diseased airways, demonstrating productive exposure of the immune system to staphylococcal products. SE-IgE has been shown to be a biomarker for severe disease, and also to predict disease progression [44]. SE-IgE in the airways is functional, as it has been demonstrated to rapidly degranulate mast cells and basophils upon contact with the specific antigen, functioning as allergen in this situation, and to facilitate antigen binding (FAB assay) to B cells $[48,70]$ in CRSwNP. SE-IgE measured within CRSwNP tissue is associated with 


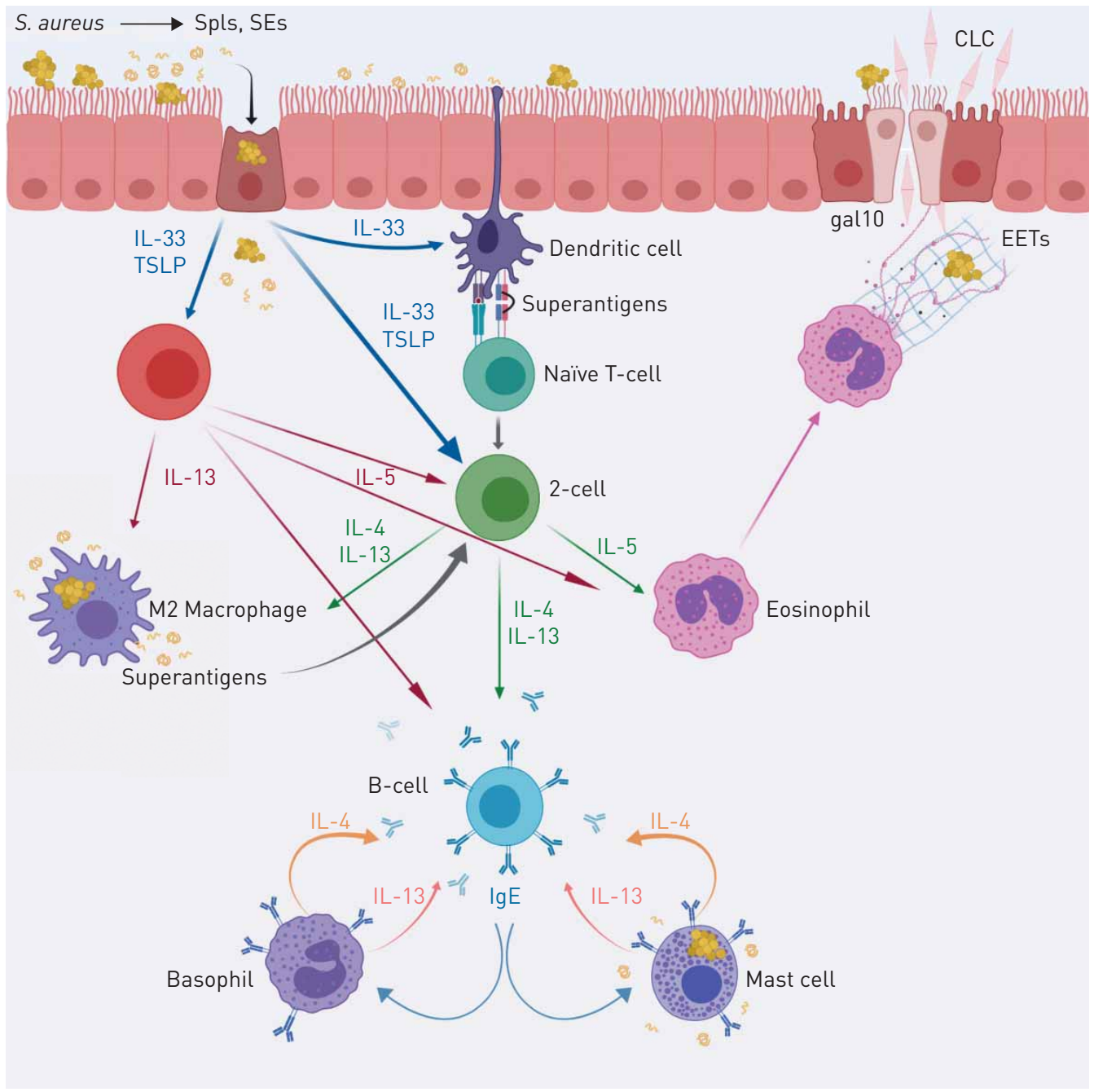

FIGURE 2 Summary of impacts of Staphylococcus aureus and its proteins on the human airway immune response. S. aureus colonises the airway epithelium, and induces the release of interleukin (IL)-33 and thymic stromal lymphopoietin (TSLP) [60]. These recruit and activate innate lymphocyte cells (ILC)2s to release IL-5 and IL-13 [24], activating T helper (Th) 2 cells and macrophages; those host S. aureus intracellularly [49], releasing proteins such as serine protease like proteins (spls) and T-cell superantigens (SES) [24], and further trigger T-cells [87, 89]. IL-33 also activates dendritic cells (DCs), which may via superantigens prime naive T-cells to develop into Th2 cells. IL-4 and IL-13 then activate B-cells and initiate polyclonal immunoglobulin (Ig)E production, further supported by mast cells and basophils. Also mast cells have been shown to host intracellular S. aureus. IL-5 released by ILC2s or TH2 cells activate eosinophils, which upon exposure to $S$. aureus migrate to the epithelium and release extracellular eosinophilic traps (EETs) [50], consisting of major basic protein, DNA and galectin 10 (gal10). Gal10 crystalises to Charcot-Leyden-Crystals (CLCs), leading to persistent stimulation of the respiratory epithelium [51].

asthma comorbidity and recurrence of disease, as well as severe type 2 immune inflammation [98]. Europe-wide SE-IgE is associated with asthma [26], and independent studies in patients cohorts with severe asthma, the marker was correlated with asthma severity and its clinical expressions, such as hospitalisation, systemic corticosteroid usage and impaired lung function [23]. Recently, in the EGEA cohort, SE-IgE was again demonstrated to be associated with disease severity, but for the first time it was also shown to be a significant risk factor to have moderate-severe asthma about a decade later [44]; moreover, being sensitised to SES was a risk factor for having asthma exacerbations years later, whereas a positive SPT to house dust mite did not contribute to that risk. To date SE-IgE thus was the only biomarker to predict the development of severe disease. In atopic dermatitis, skin colonisation with $S$. aureus is significantly increased versus healthy subjects, reaching up to $90 \%$ of atopic dermatitis patients, and SE-IgE levels are again associated with severe disease $[99,100]$. A possible approach to reduce SE-IgE may be based on anti-IgE strategies, such as omalizumab, which has been demonstrated to be effective in SE-IgE positive, allergic and non-allergic CRSwNP and "non-allergic" asthma; [101] however, its efficacy in "SE-IgE only" positive subjects with severe airway disease without further sensitizations remains to be shown. 
S. aureus, belonging to the microbiome of house dust mites, also was found in house dust samples, similar to other bacteria, and could be cultured from it [29,30]. Furthermore, S. aureus-derived extracellular vesicles are present in the environment [28], and specific staphylococcal proteins ( $\mathrm{SEA}, \mathrm{SEB}$ and $\mathrm{SEC}$ ) have been found in about $50 \%$ of dust samples from houses of asthmatic subjects and have been associated with asthma [29]. Sensitisations to house dust mites and bacterial antigens often occur together in atopic dermatitis subjects, indicating that, like HDM allergens, also staphylococcal allergens induce specific IgE antibodies; again, IgE sensitisation to bacterial antigens but not to HDM allergens was associated with severe atopic dermatitis [30].

\section{Future research needs}

However, we need to further dissect the pathophysiological relevance of the different functions of $S$. aureus proteins, from superantigens, superallergens to sole allergens, specifically to estimate the impact on humans when exposed to environmental S. aureus proteins. Does SE-IgE in the serum of an asthmatic patient predict allergic reactions upon contact with the allergen? The main obstacles for skin testing are the toxic effects of SES, which are potent T-cell mitogens, precluding their use in humans. As SES are very stable, recalcitrant to heat inactivation, their superantigenicity has to be eliminated by gene engineering. The structure function relationships of several SES were already clarified in the 1990s. On that basis inactivating mutations have been predicted and experimentally confirmed for a number of staphylococcal SAgs $[73,102]$. Detoxified SEB has been generated by introducing point mutations for use as an anti-SEB vaccine. Such reagents have already successfully passed a clinical phase I study, demonstrating safety and immunogenicity [103]. Detoxified ${ }_{\mathrm{SEB}} \mathrm{B}$ could first be used in human tissue models to show the allergenic properties, followed by skin prick and nasal/bronchial provocation tests just like those performed with other inhalant allergens. Correlating the results of the in vivo tests with measurements of serum SE-IgE by immunoassay systems would reveal the latter's diagnostic capacity such that skin testing might later be avoided in severe asthmatics. A similar research programme should be focussed on S. aureus Spls and extended in perspective to the entire allergome of the species. The SEs and the other $S$. aureus allergens could then be added to the allergologists' diagnostic and therapeutic armamentarium to improve the fate especially of "non-allergic" patients with severe asthma, presenting with high total serum IgE without evidence of sensitisation to HDM or other common inhalant allergens.

Antibiotics and oral corticosteroids unfortunately only have temporary effects on this disease, unless given long term, which increases the risk for adverse events; the impact on SE-IgE therefore is unknown. For therapy, detoxified SES are in development for vaccination in the future, which might impact on the allergenic, but also on the superantigenic properties of SES. Another approach in SE-IgE positive subjects may be anti-IgE treatment, as discussed above; as studies in severe CRSwNP and asthma with Omalizumab have already been performed, the further analysis of such patient's IgE pattern may easily provide a perspective, comparing the efficacy of the drug in SE-IgE only positive subjects to placebo treated and the whole group of verum treated subjects. If effective, SE-IgE should be added to the allergens regularly tested in severe asthma patients, offering a new treatment option to asthmatics formerly considered "non-atopic".

\section{Summary}

In summary, several groups of staphylococcal proteins are able to interact with the human airway mucosa and its immune system, including the epithelium, ILCs, dendritic cells, T and B cells, mast cells, basophils and eosinophils. Some of these interactions are shared by other bacteria or infectious agents, such as the release of IL-33 from the epithelium; others are specific to the superantigenic properties of the S. aureus proteome. Using the human nasal mucosa as a surrogate for bronchial mucosa, the pathomechanisms used by $S$. aureus to induce asthma have been partially deciphered in the human, not only in mice. In large epidemiological studies as well as in smaller cohorts, the association of SE-IgE with upper and lower airway disease, and severity of CRSwNP and asthma, has been demonstrated repeatedly in independent populations and studies. These studies clearly show that SE-IgE is associated with disease severity in asthma, atopic dermatitis and CRSwNP, and predicts severe disease prospectively.

Conflict of interest: C. Bachert has nothing to disclose. M. Humbert reports personal fees from AstraZeneca, GSK, Merck, Novartis, Roche, Sanofi and Teva, outside the submitted work. N.A. Hanania has received honoraria for serving on advisory boards or as consultant from Novartis, Genentech, Roche, Novartis, AstraZeneca, GSK, Gossamer Bio and Boehringer Ingelheim; his institution received research grant support on his behalf from GSK, Boehringer Ingelheim and AstraZeneca. N. Zhang has nothing to disclose. S. Holgate has nothing to disclose. R. Buhl has nothing to disclose. B.M. Bröker has nothing to disclose.

Support statement: C. Bachert: this work was supported by FWO Flanders, EOS project GOG2318N, and grants from FWO Flanders (G065319N, 1506218N, 1507118N, G051918N, G065319N 1515516N, 1841713N, G.039412N, 
G.067512N and FWO/PDO/108), the Interuniversity Attraction Poles Grant P7/30, from Ghent University BOF 14-GOA-019 and BOF 01/03618, and the European Commission's Seventh Framework Programme number 260895 (PREDICTA). B.M. Bröker: this work was supported by the German Research Foundation (CRC-TRR 34, RTG 1870), the Federal Ministry of Research and Education (InfectControl 2020), the EU (IMI-Combacte) as well as the State of Mecklenburg Western Pomerania and the European Social Fund (ESF; Card-ii-Omics, KoInfekt). Funding information for this article has been deposited with the Crossref Funder Registry.

\section{References}

1 Hew M, Lee L, Susanto NH, et al. The 2016 Melbourne thunderstorm asthma epidemic: Risk factors for severe attacks requiring hospital admission. Allergy 2019; 74: 122-130.

2 Brusselle GG, Maes T, Bracke KR. Eosinophils in the spotlight: Eosinophilic airway inflammation in nonallergic asthma. Nat Med 2013; 19: 977-979.

3 Barnes PJ. Intrinsic asthma: not so different from allergic asthma but driven by superantigens? Clin Exp Allergy 2009; 39: 1145-1151.

4 Humbert M, Durham SR, Ying S, et al. IL-4 and IL-5 mRNA and protein in bronchial biopsies from patients with atopic and nonatopic asthma: evidence against "intrinsic" asthma being a distinct immunopathologic entity. Am J Respir Crit Care Med 1996; 154: 1497-1504.

5 Kotsimbos TC, Ghaffar O, Minshall EM, et al. Expression of the IL-4 receptor alpha-subunit is increased in bronchial biopsy specimens from atopic and nonatopic asthmatic subjects. J Allergy Clin Immunol 1998; 102: 859-866.

6 Humbert M, Menz G, Ying S, et al. The immunopathology of extrinsic (atopic) and intrinsic (non-atopic) asthma: more similarities than differences. Immunol Today 1999; 20: 528-533.

7 Schreiber J, Mailaender C. The Identify Project: do we know the most prevalent allergens in severe asthma? Am J Respir Crit Care Med 2018; 197: A2493.

8 Schreiber J, Bröker BM, Ehmann R, et al. Non-atopic severe asthma might not always be non-atopic: Sensitization towards Staphylococcus aureus enterotoxins A and B and other allergens. J Allergy Clin Immunol 2019; 143: 2279-2280.

9 de Groot JC, Ten Brinke A, Bel EH. Management of the patient with eosinophilic asthma: a new era begins. ERJ Open Res 2015; 1: 00024-2015.

10 Fokkens WJ, Lund VJ, Mullol J, et al. EPOS 2012: European position paper on rhinosinusitis and nasal polyps 2012. A summary for otorhinolaryngologists. Rhinology 2012; 50: 1-12.

11 Bachert C, Zhang N. Chronic rhinosinusitis and asthma: novel understanding of the role of IgE 'above atopy'. J Intern Med 2012; 272: 133-143.

12 Chung KF. Asthma phenotyping: a necessity for improved therapeutic precision and new targeted therapies. J Intern Med 2016; 279: 192-204.

13 Garcia G, Magnan A, Chiron R, et al. A proof-of-concept, randomized, controlled trial of omalizumab in patients with severe, difficult-to-control, nonatopic asthma. Chest 2013; 144: 411-419.

14 Gevaert $\mathrm{P}$, Nouri-Aria $\mathrm{KT}$, Wu $\mathrm{H}$, et al. Local receptor revision and class switching to IgE in chronic rhinosinusitis with nasal polyps. Allergy 2013; 68: 55-63.

15 Lowy F. Staphylococcus aureus infections. N Engl J Med 1998; 339: 520-529.

16 Tong SY, Davis JS, Eichenberger E, et al. Staphylococcus aureus infections: epidemiology, pathophysiology, clinical manifestations, and management. Clin Microbiol Rev 2015; 28: 603-661.

17 Sollid JU, Furberg AS, Hanssen AM, et al. Staphylococcus aureus: determinants of human carriage. Infect Genet Evol 2014; 21: 531-541.

18 de Benito S, Alou L, Becerro-de-Bengoa-Vallejo R, et al. Prevalence of Staphylococcus spp. nasal colonization among doctors of podiatric medicine and associated risk factors in Spain. Antimicrob Resist Infect Control 2018; 7: 24 .

19 Holtfreter S, Kolata J, Bröker BM. Towards the immune proteome of Staphylococcus aureus - The anti-S. aureus antibody response. Int J Med Microbiol 2010; 300: 176-192.

20 Brown AF, Murphy AG, Lalor SJ, et al. Memory Th1 cells are protective in invasive Staphylococcus aureus infection. PLoS Pathog 2015; 11: e1005226.

21 Kolata JB, Kühbandner I, Link C, et al. The fall of a dogma? unexpected high t-cell memory response to Staphylococcus aureus in humans. J Infect Dis 2015; 212: 830-838.

22 Bröker BM, Mrochen D, Péton V. The T cell response to Staphylococcus aureus. Pathogens 2016; 5: 31.

23 Bachert C, van Stehen K, Zhang N, et al. Specific IgE against Staphylococcus aureus enterotoxins: an independent risk factor for asthma. J Allergy Clin Immunol 2012; 130: 376-381.

24 Teufelberger AR, Nordengrün M, Braun H, et al. The IL-33/ST2 axis is crucial in type 2 airway responses induced by Staphylococcus aureus-derived serine protease-like protein D. J Allergy Clin Immunol 2018; 141: 549-559.

25 Nordengrün M, Michalik S, Völker U, et al. The quest for bacterial allergens. Int J Med Microbiol 2018; 308: 738-750.

26 Tomassen P, Jarvis D, Newson R, et al. Staphylococcus aureus enterotoxin-specific IgE is associated with asthma in the general population: a GA(2)LEN study. Allergy 2013; 68: 1289-1297.

27 Bachert C, Gevaert P, Holtappels G, et al. Total and specific IgE in nasal polyps is related to local eosinophilic inflammation. J Allergy Clin Immunol 2001; 107: 607-614.

28 Yang J, Kim YK, Kang TS, et al. Importance of indoor dust biological ultrafine particles in the pathogenesis of chronic inflammatory lung diseases. Environ Health Toxicol 2017; 32: e2017021.

29 Davis MF, Ludwig S, Brigham EP, et al. Effect of home exposure to Staphylococcus aureus on asthma in adolescents. J Allergy Clin Immunol 2018; 141: 402-405.

30 Dzoro S, Mittermann I, Resch-Marat Y, et al. House dust mites as potential carriers for IgE sensitization to bacterial antigens. Allergy 2018; 73: 115-124.

31 Ludwig S, Jimenez-Bush I, Brigham E, et al. Analysis of home dust for Staphylococcus aureus and staphylococcal enterotoxin genes using quantitative PCR. Sci Total Environ 2017; 581-582: 750-755. 
Wertheim HF, Mellesv DC, Vos MC, et al. The role of nasal carriage in Staphylococcus aureus infections. Lancet Infect Dis 2015; 5: 751-762.

Kim YC, Won HK, Lee JW, et al. Staphylococcus aureus nasal colonization and asthma in adults: systematic review and meta-analysis. J Allergy Clin Immunol Pract 2018; 7: 606-615.

Van Zele T, Gevaert P, Watelet JB, et al. Staphylococcus aureus colonization and IgE antibody formation to enterotoxins is increased in nasal polyposis. J Allergy Clin Immunol 2014; 114: 981-983.

Corriveau MN, Zhang N, Holtappels G, et al. Detection of Staphylococcus aureus in nasal tissue with peptide nucleic acid-fluorescence in situ hybridization. Am J Rhinol Allergy 2009; 23: 461-465.

Schmidt F, Meyer T, Sundaramoorthy N, et al. Characterization of human and Staphylococcus aureus proteins in respiratory mucosa by in vivo- and immunoproteomics. J Proteomics 2017; 155: 31-39.

Bachert C, Claeys SE, Tomassen P, et al. Rhinosinusitis and asthma: a link for asthma severity. Curr Allergy Asthma Rep 2010; 10: 194-201.

Tomassen P, Vandeplas G, Van Zele T, et al. Inflammatory endotypes of chronic rhinosinusitis based on cluster analysis of biomarkers. J Allergy Clin Immunol 2016; 137: 1449-1456.

Kowalski ML, Cieślak M, Pérez-Novo CA, et al. Clinical and immunological determinants of severe/refractory asthma (SRA): association with Staphylococcal superantigen-specific IgE antibodies. Allergy 2011; 66: 32-38.

Matsumoto H, Kanemitsu Y, Nagasaki T, et al. Staphylococcus aureus enterotoxin sensitization involvement and its association with the CysLTR1 variant in different asthma phenotypes. Ann Allergy Asthma Immunol 2017; 118: 197-203.

Sorensen M, Klingenberg C, Wickman M, et al. Staphylococcus aureus enterotoxin sensitization is associated with allergic poly-sensitization and allergic multimorbidity in adolescents. Allergy 2017; 72: 1548-1555.

Song WJ, Sintobin I, Sohn KH, et al. Staphylococcal enterotoxin IgE sensitization in late-onset severe eosinophilic asthma in the elderly. Clin Exp Allergy 2016; 46: 411-421.

Rohde G, Gevaert P, Holtappels G, et al. Increased IgE-antibodies to Staphylococcus aureus enterotoxins in patients with COPD. Respir Med 2004; 98: 858-864.

Sintobin I, Siroux V, Holtappels G, et al. Sensitisation to staphylococcal enterotoxins and asthma severity: a longitudinal study in the EGEA cohort. Eur Respir J 2019; 54: 1900198.

Berube BJ, Bubeck Wardenburg J. Staphylococcus aureus alpha-toxin: nearly a century of intrigue. Toxins (Basel) 2013; 5: 1140-1166.

Nakamura Y, Oscherwitz J, Cease KB, et al. Staphylococcus $\delta$-toxin induces allergic skin disease by activating mast cells. Nature 2013; 503: 397-401.

Patou J, Gevaert P, Van Zele T. Staphylococcus aureus enterotoxin B, protein A, and lipoteichoic acid stimulations in nasal polyps. J Allergy Clin Immunol 2018; 121: 110-115.

Zhang N, Holtappels G, Gevaert P, et al. Mucosal tissue polyclonal IgE is functional in response to allergen and SEB. Allergy 2011; 66: 141-148.

Bachert C, Holtappels G, Merabishvili M, et al. Staphylococcus aureus controls interleukin-5 release in upper airway inflammation. J Proteomics 2018; 180: 53-60.

Gevaert E, Zhang N, Krysko O, et al. Extracellular eosinophilic traps in association with Staphylococcus aureus at the site of epithelial barrier defects in patients with severe airway inflammation. J Allergy Clin Immunol 2017; 139: $1849-1860$.

Persson EK, Verstraete K, Heyndrickx I, et al. Protein crystallization promotes type 2 immunity and is reversible by antibody treatment. Science 2019; 364: eaaw4295.

Kato A, Peters A, Suh L, et al. Evidence of a role for B cell-activating factor of the TNF family in the pathogenesis of chronic rhinosinusitis with nasal polyps. J Allergy Clin Immunol 2008; 121: 1385-1392.

Huvenne W, Callebaut I, Plantinga M, et al. Staphylococcus aureus enterotoxin B facilitates allergic sensitization in experimental asthma. Clin Exp Allergy 2010; 40: 1079-1090.

Graille M, Stura EA, Corper AL, et al. Crystal structure of a Staphylococcus aureus protein A domain complexed with the Fab fragment of a human IgM antibody: structural basis for recognition of B-cell receptors and superantigen activity. Proc Natl Acad Sci USA 2000; 97: 5399-5404.

Bröker BM, Holtfreter S, Bekeredjian-Ding I. Immune control of Staphylocuccus aureus - Regulation and counter-regulation of the adaptive immune response. Int J Med Microbiol 2014; 304: 204-214.

Wills-Karp M, Lewkowich IP. Immunologic mechanism of allergic disorders. Fundamental Immunology 2013. W. E. Paul. Philadelphia, Lippincott Williams \& Wilkins: 1113-1153.

Zielinski CE, Corti D, Mele F, et al. Dissecting the human immunologic memory for pathogens. Immunol Rev 2011; 240: 40-51.

Reed SB, Wesson CA, Liou LE, et al. Molecular characterization of a novel Staphylococcus aureus serine protease operon. Infect Immun 2001; 69: 1521-1527.

Zdzalik M, Karim AY, Wolski K, et al. Prevalence of genes encoding extracellular proteases in Staphylococcus aureus - important targets triggering immune response in vivo. FEMS Immunol Med Microbiol 2012; 66: 220-229.

Feng L, Zhang N, Holtappels G, et al. Staphylococcus aureus induces a mucosal type 2 immune response via epithelial cell-derived cytokines. Am J Respir Crit Care Med 2019; 198: 452-463.

Stach N, Kalinska M, Zdzalik M, et al. Unique substrate specificity of SplE serine protease from Staphylococcus aureus. Structure 2018; 26: 572-579.

Van Crombruggen K, Taveirne S, Holtappels G, et al. Innate lymphoid cells in the upper airways: importance of CD117 and IL-1RI expression. Eur Respir J 2018; 52: 1800742.

Hoyler T, Klose CS, Souabni A, et al. The transcription factor GATA-3 controls cell fate and maintenance of type 2 innate lymphoid cells. Immunity 2012; 37: 634-648.

Mjösberg J, Bernink J, Golebski K, et al. The transcription factor GATA3 is essential for the function of human type 2 innate lymphoid cells. Immunity 2012; 37: 649-659.

Hazenberg MD, Spits H. Human innate lymphoid cells. Blood 2014; 124: 700-709.

Mazzurana L, Rao A, Van Acker A, et al. The roles for innate lymphoid cells in the human immune system. Semin Immunopathol 2018; 40: 407-419.

Sonnenberg GF, Hepworth MR. Functional interactions between innate lymphoid cells and adaptive immunity. Nat Rev Immunol 2019; 19: 599-613. 
Duerr CU, McCarthy CD, Mindt BC, et al. Type I interferon restricts type 2 immunopathology through the regulation of group 2 innate lymphoid cells. Nat Immunol 2016; 17: 65-75.

Cheng KJ, Wang SQ, Xu YY. Different roles of Staphylococcus aureus enterotoxin in different subtypes of nasal polyps. Exp Ther Med 2017; 13: 321-326.

Shamji M, Thomsen I, Layhadi J, et al. Broad immunglobulin G repertoire in chronic rhinosinusitis with nasal polyps regulates pro-inflammatory IgE responses. J Allergy Clin Immunol 2019; 143: 2086-2094.

Shamji MH, Kappen J, Abubakar-Waziri H, et al. Nasal allergen-neutralizing IgG4 antibodies block IgE-mediated responses: Novel biomarker of subcutaneous grass pollen immunotherapy. J Allergy Clin Immunol 2019; 143: 1067-1076.

Fraser JD, Proft T. The bacterial superantigen and superantigen-like proteins. Immunol Rev 2018; 225: 226-243.

Spaulding AR, Salgado-Pabon W, Kohler PL, et al. Staphylococcal and streptococcal superantigen exotoxins. Clin Microbiol Rev 2013; 26: 422-447.

Grumann D, Nübel U, Bröker BM. Staphylococcus aureus toxins-their functions and genetics. Infect Genet Evol 2014; 21: 583-592.

Holtfreter S, Grumann D, Schmudde M, et al. Clonal distribution of superantigen genes in clinical Staphylococcus aureus isolates. J Clin Microbiol 2007; 45: 2669-2680. J Infect Dis 2004; 189: 2334-2336. specific for their colonizing strain: a potential explanation for their improved prognosis in severe sepsis. J Infect Dis 2006; 193: 1275-1278.

Bachert C, Gevaert P, Zhang N, et al. Role of staphylococcal superantigens in airway disease. Chem Immuno Allergy 2007; 93: 214-236.

Thammavongsa V, Kim HK, Missiakas D, et al. Staphylococcal manipulation of host immune responses. Nat Rev Microbiol 2015; 13: 529-543.

Strommenger B, Braulke C, Heuck D, et al. spa Typing of Staphylococcus aureus as a frontline tool in epidemiological typing. J Clin Microbiol 2018; 46: 574-581.

Davies AM, Sutton BJ. Human IgG4: a structural perspective. Immunol Rev 2015; 268: 139-159.

Stentzel S, Teufelberger A, Nordengrün M, et al. Spls: Staphylococcus serine protease-like proteins are pacemakers of allergic airway reactions. J Allergy Clin Immunol 2017; 139: 492-500.

Lua WH, Su ST, Yeo JY, et al. Role of the IgE variable heavy chain in FcepsilonRIalpha and superantigen binding in allergy and immunotherapy. J Allergy Clin Immunol 2019; 144: 514-523.

Pauli NT, Kim HK, Falugi F, et al. Staphylococcus aureus infection induces protein A-mediated immune evasion in humans. J Exp Med 2014; 211: 2331-2339.

Thammavongsa V, Rauch S, Kim HK, et al. Protein A-neutralizing monoclonal antibody protects neonatal mice against Staphylococcus aureus. Vaccine 2015; 33: 523-526.

Chen X, Sun Y, Missiakas D, et al. Staphylococcus aureus decolonization of mice with monoclonal antibody neutralizing protein A. J Infect Dis 2019; 219: 884-888.

Genovese A, Bouvet JP, Florio G, et al. Bacterial immunoglobulin superantigen proteins A and L activate human heart mast cells by interacting with immunoglobulin E. Infect Immun 2000; 68: 5517-5524.

Kristiansen SV, Pascual V, Lipsky PE. Staphylococcal protein A induces biased production of Ig by VH3-expressing B lymphocytes. J Immunol 1994; 153: 2974-2982.

Levinson AI, Kozlowski L, Zheng Y, et al. B-cell superantigens: definition and potential impact on the immune response. J Clin Immunol 1995; 15: 26S-36S.

Goodyear CS, Silverman GJ. B cell superantigens: a microbe's answer to innate-like B cells and natural antibodies. Springer Semin Immunopathol 2005; 26: 463-484.

Silverman GJ, Goodyear CS. Confounding B-cell defences: lessons from a staphylococcal superantigen. Nat Rev Immunol 2006; 6: 465-475.

Pryjma J, Munoz J, Virella G, et al. Evaluation of $\operatorname{IgM}$, IgG, IgA, IgD, and IgE secretion by human peripheral blood lymphocytes in cultures stimulated with pokeweed mitogen and Staphylococcus aureus Cowan I. Cell Immunol 1980; 50: 115-124.

Hemady Z, Blomberg F, Gellis S, et al. IgE production in vitro by human blood mononuclear cells: a comparison between atopic and nonatopic subjects. J Allergy Clin Immunol 1983; 71: 324-330.

Del Prete G, Maggi E, Romagnani S, et al. Human IgE biosynthesis in vitro. Clin Rev Allergy 1989; 7: 193-216.

Krysko O, Teufelberger A, Van Nevel S, et al. Protease/antiprotease network in allergy: The role of Staphylococcus aureus protease-like proteins. Allergy 2019; 19: 2077-2086.

Eberl G, Colonna M, Di Santo JP, et al. Innate lymphoid cells. Innate lymphoid cells: a new paradigm in immunology. Science 2015; 348: aaa6566.

Trends Mol Med 2019; 25: 696-707.

Bachert C, Zhang N, Holtappels G, et al. Presence of IL-5 protein and IgE-antibodies to staphylococcal enterotoxins in nasal polyps is associated with co-morbid asthma. J Allergy Clin Immunol 2010; 126: 962-968.

Nowicka D, Grywalska E. The role of immune defects and colonization of Staphylococcus aureus in the pathogenesis of atopic dermatitis. Anal Cell Pathol (Amst) 2018; 2018: 1956403.

Weidinger S, Beck LA, Bieber T, et al. Atopic dermatitis. Nat Rev Dis Primers 2018; 4: 1.

Humbert M, Bousquet J, Bachert C, et al. IgE-mediated multimorbidities in allergic asthma and the potential for omalizumab therapy. J Allergy Clin Immunol Pract 2019; 7: 1418-1429.

Aman MJ. Superantigens of a superbug: major culprits of Staphylococcus aureus disease? Virulence 2017; 8: 607-610.

Chen WH, Pasetti MF, Adhikari RP, et al. Safety and Immunogenicity of a Parenterally Administered, Structure-Based Rationally Modified Recombinant Staphylococcal Enterotoxin B Protein Vaccine, STEBVax. Clin Vaccine Immunol 2016; 23: 918-925. 